\title{
Wave Patterns in Spatial Games and the Evolution of Cooperation
}

Régis Ferrière (rferrier@hall.snv.jussieu.fr)

Richard E.Michod (michod@ccit.arizona.edu)

\section{Approved by}

Ulf Dieckmann (dieckman@iiasa.ac.at)

Project Coordinator, Adaptive Dynamics Network

December 1999 


\section{IIASA STUDIES IN ADAPTIVE DYNAMICS No.42}

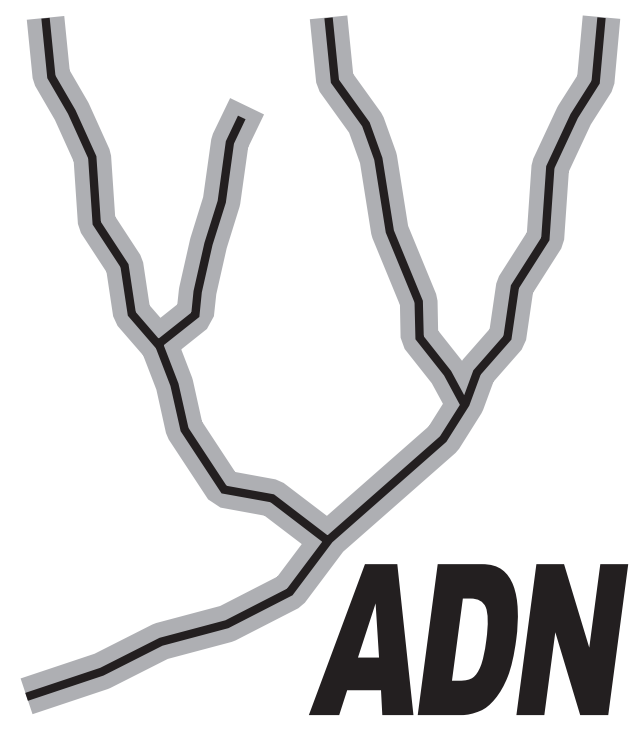

The Adaptive Dynamics Network at IIASA fosters the development of new mathematical and conceptual techniques for understanding the evolution of complex adaptive systems.

Focusing on these long-term implications of adaptive processes in systems of limited growth, the Adaptive Dynamics Network brings together scientists and institutions from around the world with IIASA acting as the central node.

Scientific progress within the network is reported in the IIASA Studies in Adaptive Dynamics series.

\section{THE ADAPTIVE DYNAMICS NETWORK}

The pivotal role of evolutionary theory in life sciences derives from its capability to provide causal explanations for phenomena that are highly improbable in the physicochemical sense. Yet, until recently, many facts in biology could not be accounted for in the light of evolution. Just as physicists for a long time ignored the presence of chaos, these phenomena were basically not perceived by biologists.

Two examples illustrate this assertion. Although Darwin's publication of “The Origin of Species" sparked off the whole evolutionary revolution, oddly enough, the population genetic framework underlying the modern synthesis holds no clues to speciation events. A second illustration is the more recently appreciated issue of jump increases in biological complexity that result from the aggregation of individuals into mutualistic wholes.

These and many more problems possess a common source: the interactions of individuals are bound to change the environments these individuals live in. By closing the feedback loop in the evolutionary explanation, a new mathematical theory of the evolution of complex adaptive systems arises. It is this general theoretical option that lies at the core of the emerging field of adaptive dynamics. In consequence a major promise of adaptive dynamics studies is to elucidate the long-term effects of the interactions between ecological and evolutionary processes.

A commitment to interfacing the theory with empirical applications is necessary both for validation and for management problems. For example, empirical evidence indicates that to control pests and diseases or to achieve sustainable harvesting of renewable resources evolutionary deliberation is already crucial on the time scale of two decades.

The Adaptive Dynamics Network has as its primary objective the development of mathematical tools for the analysis of adaptive systems inside and outside the biological realm. 


\section{IIASA STUDIES IN ADAPTIVE DYNAMICS}

No. 1 Metz JAJ, Geritz SAH, Meszéna G, Jacobs FJA, van Heerwaarden JS:

Adaptive Dynamics: A Geometrical Study of the Consequences of Nearly Faithful Reproduction.

IIASA Working Paper WP-95-099.

In: van Strien SJ, Verduyn Lunel SM (eds.): Stochastic and Spatial Structures of Dynamical Systems, Proceedings of the Royal Dutch Academy of Science (KNAW Verhandelingen), North Holland, Amsterdam, pp. 183-231 (1996).

No. 2 Dieckmann U, Law R:

The Dynamical Theory of Coevolution: A Derivation from Stochastic

Ecological Processes.

IIASA Working Paper WP-96-001.

Journal of Mathematical Biology (1996) 34, 579-612.

No. 3 Dieckmann U, Marrow P, Law R:

Evolutionary Cycling of Predator-Prey Interactions: Population Dynamics and the Red Queen.

Journal of Theoretical Biology (1995) 176, 91-102.

No. 4 Marrow P, Dieckmann U, Law R:

Evolutionary Dynamics of Predator-Prey Systems: An Ecological

Perspective.

IIASA Working Paper WP-96-002.

Journal of Mathematical Biology (1996) 34, 556-578.

No. 5 Law R, Marrow P, Dieckmann U:

On Evolution under Asymmetric Competition.

IIASA Working Paper WP-96-003.

Evolutionary Ecology (1997) 11, 485-501.

No. 6 Metz JAJ, Mylius SD, Diekmann O:

When Does Evolution Optimise? On the Relation between Types of Density

Dependence and Evolutionarily Stable Life History Parameters.

IIASA Working Paper WP-96-004.

No. 7 Ferrière R, Gatto M:

Lyapunov Exponents and the Mathematics of Invasion in Oscillatory or Chaotic Populations.

Theoretical Population Biology (1995) 48, 126-171.

No. 8 Ferrière R, Fox GA:

Chaos and Evolution.

Trends in Ecology and Evolution (1995) 10, 480-485. 
No. 9 Ferrière R, Michod RE:

The Evolution of Cooperation in Spatially Heterogeneous Populations.

IIASA Working Paper WP-96-029.

American Naturalist (1996) 147, 692-717.

No. 10 Van Dooren TJM, Metz JAJ:

Delayed Maturation in Temporally Structured Populations with Non-Equilibrium Dynamics.

IIASA Working Paper WP-96-070.

Journal of Evolutionary Biology (1998) 11, 41-62.

No. 11 Geritz SAH, Metz JAJ, Kisdi E, Meszéna G:

The Dynamics of Adaptation and Evolutionary Branching.

IIASA Working Paper WP-96-077.

Physical Review Letters (1997) 78, 2024-2027.

No. 12 Geritz SAH, Kisdi E, Meszéna G, Metz JAJ:

Evolutionarily Singular Strategies and the Adaptive Growth and Branching of the Evolutionary Tree.

IIASA Working Paper WP-96-114.

Evolutionary Ecology (1998) 12, 35-57.

No. 13 Heino M, Metz JAJ, Kaitala V:

Evolution of Mixed Maturation Strategies in Semelparous Life-Histories: the

Crucial Role of Dimensionality of Feedback Environment.

IIASA Working Paper WP-96-126.

Philosophical Transactions of the Royal Society of London Series B (1997) 352, 1647 1655.

No. 14 Dieckmann U:

Can Adaptive Dynamics Invade?

IIASA Working Paper WP-96-152.

Trends in Ecology and Evolution (1997) 12, 128-131.

No. 15 Meszéna G, Czibula I, Geritz SAH:

Adaptive Dynamics in a Two-Patch Environment: a Simple Model for

Allopatric and Parapatric Speciation.

IIASA Interim Report IR-97-001.

Journal of Biological Systems (1997) 5, 265-284.

No. 16 Heino M, Metz JAJ, Kaitala V:

The Enigma of Frequency-Dependent Selection.

IIASA Interim Report IR-97-061.

Trends in Ecology and Evolution (1998) 13, 367-370.

No. 17 Heino M:

Management of Evolving Fish Stocks.

IIASA Interim Report IR-97-062.

Canadian Journal of Fisheries and Aquatic Sciences (1998) 55, 1971-1982. 
No. 18 Heino M:

Evolution of Mixed Reproductive Strategies in Simple Life-History Models.

IIASA Interim Report IR-97-063.

No. 19 Geritz SAH, van der Meijden E, Metz JAJ:

Evolutionary Dynamics of Seed Size and Seedling Competitive Ability.

IIASA Interim Report IR-97-071.

Theoretical Population Biology (1999) 55, 324-343.

No. 20 Galis F, Metz JAJ:

Why are there so many Cichlid Species? On the Interplay of Speciation and Adaptive Radiation.

IIASA Interim Report IR-97-072.

Trends in Ecology and Evolution (1998) 13, 1-2.

No. 21 Boerlijst MC, Nowak MA, Sigmund K:

Equal Pay for all Prisoners. /The Logic of Contrition.

IIASA Interim Report IR-97-073.

AMS Monthly (1997) 104, 303-307.

Journal of Theoretical Biology (1997) 185, 281-294.

No. 22 Law R, Dieckmann U:

Symbiosis without Mutualism and the Merger of Lineages in Evolution.

IIASA Interim Report IR-97-074.

Proceedings of the Royal Society of London Series B (1998) 265, 1245-1253.

No. 23 Klinkhamer PGL, de Jong TJ, Metz JAJ:

Sex and Size in Cosexual Plants.

IIASA Interim Report IR-97-078.

Trends in Ecology and Evolution (1997) 12, 260-265.

No. 24 Fontana W, Schuster P:

Shaping Space: The Possible and the Attainable in RNA Genotype-Phenotype Mapping.

IIASA Interim Report IR-98-004.

Journal of Theoretical Biology (1998) 194, 491-515.

No. 25 Kisdi E, Geritz SAH:

Adaptive Dynamics in Allele Space: Evolution of Genetic Polymorphism by Small Mutations in a Heterogeneous Environment.

IIASA Interim Report IR-98-038.

No. 26 Fontana W, Schuster P:

Continuity in Evolution: On the Nature of Transitions.

IIASA Interim Report IR-98-039.

Science (1998) 280, 1451-1455. 
No. 27 Nowak MA, Sigmund K:

Evolution of Indirect Reciprocity by Image Scoring. / The Dynamics of

Indirect Reciprocity.

IIASA Interim Report IR-98-040.

Nature (1998) 393, 573-577.

Journal of Theoretical Biology (1998) 194, 561-574.

No. 28 Kisdi E:

Evolutionary Branching Under Asymmetric Competition.

IIASA Interim Report IR-98-045.

Journal of Theoretical Biology (1999) 197, 149-162.

No. 29 Berger U:

Best Response Adaptation for Role Games.

IIASA Interim Report IR-98-086.

No. 30 Van Dooren TJM:

The Evolutionary Ecology of Dominance-Recessivity

IIASA Interim Report IR-98-096.

Journal of Theoretical Biology (1999) 198, 519-532.

No. 31 Dieckmann U, O'Hara B, Weisser W:

The Evolutionary Ecology of Dispersal.

IIASA Interim Report IR-98-108.

Trends in Ecology and Evolution (1999) 14, 88-90.

No. 32 Sigmund K:

Complex Adaptive Systems and the Evolution of Reciprocation.

IIASA Interim Report IR-98-100.

Ecosystems (1998) 1, 444-448.

No. 33 Posch M, Pichler A, Sigmund K:

The Efficiency of Adapting Aspiration Levels.

IIASA Interim Report IR-98-103.

Proceedings of the Royal Society of London Series B (1999) 266, 1427-1435.

No. 34 Mathias A, Kisdi É:

Evolutionary Branching and Coexistence of Germination Strategies.

IIASA Interim Report IR-99-014.

No. 35 Dieckmann U, Doebeli M:

On the Origin of Species by Sympatric Speciation.

IIASA Interim Report IR-99-013.

Nature (1999) 400, 354-357.

No. 36 Metz JAJ, Gyllenberg M:

How Should We Define Fitness in Structured Metapopulation Models? Including an Application to the Calculation of Evolutionarily Stable Dispersal Strategies.

IIASA Interim Report IR-99-019. 
No. 37 Gyllenberg M, Metz JAJ:

On Fitness in Structured Metapopulations.

IIASA Interim Report IR-99-037.

No. 38 Meszéna G, Metz JAJ:

Species Diversity and Population Regulation: The Importance of Environmental Feedback Dimensionality.

IIASA Interim Report IR-99-045.

No. 39 Kisdi É, Geritz SAH:

Evolutionary Branching and Sympatric Speciation in Diploid Populations.

IIASA Interim Report IR-99-048.

No. 40 Ylikarjula J, Heino M, Dieckmann U:

Ecology and Adaptation of Stunted Growth in Fish.

IIASA Interim Report IR-99-050.

No. 41 Nowak MA, Sigmund K:

Games on Grids.

IIASA Interim Report IR-99-038.

In: Dieckmann U, Law R, Metz JAJ (eds.): The Geometry of Ecological Interactions: Simplifying Spatial Complexity, Cambridge University Press, Cambridge, UK, pp. 135150 (2000).

No. 42 Ferrière R, Michod RE:

Wave Patterns in Spatial Games and the Evolution of Cooperation.

IIASA Interim Report IR-99-041.

In: Dieckmann U, Law R, Metz JAJ (eds.): The Geometry of Ecological Interactions: Simplifying Spatial Complexity, Cambridge University Press, Cambridge, UK, pp. 318$332(2000)$.

Issues of the IIASA Studies in Adaptive Dynamics series can be obtained free of charge. Please contact:

Adaptive Dynamics Network

International Institute for Applied Systems Analysis

Schlossplatz 1

A-2361 Laxenburg

Austria

Telephone +432236 807, Telefax +432236 71313, E-Mail adn@iiasa.ac.at, Internet http://www.iiasa.ac.at/Research/ADN 


\section{Contents}

1 Introduction $\quad 1$

2 Invasion in Time- and Space-continuous Games 1

Replicator-diffusion equations . . . . . . . . . . . . . . . . . 2

Invasibility and evolutionary stability _. . . . . . . . . . . . . 3

3 Invasion of Tit For Tat in Games with Time-limited Memory 5

The spatial struggle of Tit For Tat and Always Defect . . . . . . . . . . 5

A replicator-diffusion model _. . . . . . . . . . . . . . . 6

Microscopic description of interactions. . . . . . . . . . . . 6

From microscopic interactions to macroscopic dynamics. . . . . . . . . . . 6

A spatial version of Hamilton's rule . . . . . . . . . . . . . . . . . 7

Why mobility can favor Tit For Tat . . . . . . . . . . . . . . . . 9

4 Invasion of Tit For Tat in Games with Space-limited Memory 9

Model description . . . . . . . . . . . . . . . . . . 10

Main properties of the model . . . . . . . . . . . . . . . . 10

Existence of invading waves of Tit For Tat . . . . . . . . . . . . . . . 11

5 Concluding Comments $\quad 12$ 


\title{
About the Authors
}

\author{
Régis Ferrière \\ Laboratoire d'Écologie, École Normale Supérieure \\ Paris, France \\ and \\ Adaptive Dynamics Network \\ International Institute for Applied Systems Analysis \\ Laxenburg, Austria \\ and \\ Department of Ecology and Evolutionary Biology \\ University of Arizona, Tucson AZ, USA
}

Richard E. Michod

Department of Ecology and Evolutionary Biology

University of Arizona, Tucson AZ, USA 


\title{
Wave Patterns in Spatial Games and the Evolution of Cooperation
}

\author{
Régis Ferrière \\ Richard E. Michod
}

\section{Introduction}

Our understanding of the evolution of animal behavior has been greatly enhanced by the use of game theory (Maynard Smith 1982). Classical games assume that a given individual is equally likely to interact with any other member of the population and that the success of any individual depends on the frequency of all other strategies represented in the population. Yet natural environments possess a spatial dimension: individuals have limited mobility and interact locally with their neighbors. Only recently have attempts been made to incorporate this important property into the study of evolutionary games. Different approaches have been followed: numerical simulations of "games on grids" (Nowak and May 1992; Lindgren and Nordahl 1994; see Chapter 8 in Dieckmann et al. 2000); analytical study of correlation equations for games on lattices (Nakamaru et al. 1997; see Chapter 13 in Dieckmann et al. 2000); and analytical study of "replicator-diffusion" equations (e.g., Vickers 1989; Vickers et al. 1993; Ferrière and Michod 1995, 1996; see Chapter 22 in Dieckmann et al. 2000). In this chapter we restrict ourselves to the last of these methodologies and provide an introduction to its mathematical underpinnings and biological applications. Elements of a general theory of replicator-diffusion equations are expounded in detail in articles by Vickers (1989), Hutson and Vickers (1992), Vickers et al. (1993), and Cressman and Vickers (1997). We present an overview of these important results in Section 2. Sections 3 and 4 show how replicator-diffusion models can be used to study spatial versions of the iterated Prisoner's Dilemma game, a well-known metaphor for evolution toward cooperation between genetically unrelated individuals (Trivers 1971; Axelrod and Hamilton 1981; Maynard Smith 1982; Hofbauer and Sigmund 1998).

\section{Invasion in Time- and Space-continuous Games}

When considering the adaptive dynamics of long-term evolution, the crucial question is whether a mutant phenotype can invade resident phenotypes (Metz et al. 1992; Diekmann et al. 1996; Geritz et al. 1997; Dieckmann 1997). Obviously, the very nature of an invasion event requires individual mobility, while the game theoretical context requires interactions between individuals that share the same neighborhood. This creates the need for deriving invasibility criteria that explicitly account for spatial effects in the dynamics of mutant invasion. Much of the theory of invasion in time- and space-continuous games is relatively new. This section presents the basic ideas needed for subsequent applications and points to some unsolved questions. 


\section{Replicator-diffusion equations}

Hereafter, the payoff matrix of a game is denoted by $A$. We set $A=\left[a_{i j}\right]$, where $a_{i j}$ is the payoff to an individual who plays strategy $i$ against an opponent who plays $j$. Let $Q$ be a probability vector composed of $q_{i}$ denoting the proportion of individuals who play strategy $i$. Taylor and Jonker (1978) have suggested incorporating continuous time into the dynamics of games using so-called replicator equations

$$
\frac{d q_{i}}{d t}=q_{i}\left[(A Q)_{i}-Q^{T} A Q\right] \quad(1 \leq i \leq k),
$$

where $k$ is the number of strategies, $(A Q)_{i}$ denotes the $i$ th coordinate of vector $A Q$, and the exponent $T$ indicates vector transpose. This model stems from the idea that the growth rate of a strategy is equal to its absolute payoff $(A Q)_{i}$. Hence the abundance of strategy $i$ is described by unlimited exponential growth, according to the equation $d n_{i} / d t=\left[(A Q)_{i}\right] n_{i}$. One can then write $q_{i}$ as $n_{i}$ divided by the total population density (i.e., the sum of the densities of all strategies) and use the latter equation to recover Equations (1). The replicator equation expresses the fact that the change in any strategy's frequency is determined by the population growth rate of that strategy compared with the average population growth rate, that is, the average payoff $Q^{T} A Q$. Equations (1) are important because there is a relationship between the stable equilibrium points of Equations (1) and the game's evolutionarily stable strategies, or ESSs (Zeeman 1980; Hofbauer and Sigmund 1998). [We recall that an ESS is a strategy that, when common in the population, cannot be invaded by any small group of individuals playing a different strategy; see, e.g., Maynard Smith (1982).]

The inclusion of continuous space is not so straightforward. If all individuals move at the same rate, we are on a well-worn trail (see Hadeler 1981). But it is quite reasonable to expect that the dispersal rate is affected by, or indeed part of, the strategy chosen. Using the standard diffusion approximation of a random walk (see Chapter 22 in Dieckmann et al. 2000), Vickers (1989) introduced the following "replicator-diffusion equations":

$$
\frac{\partial n_{i}}{\partial t}=n_{i}\left(\frac{(A N)_{i}}{m}-\frac{N^{T} A N}{m^{2}}\right)+\mu_{i} \frac{\partial^{2} n_{i}}{\partial x^{2}} \quad(1 \leq i \leq s),
$$

where each strategy is characterized by its own diffusion (or mobility) rate $\mu_{i}$. Here, $n_{i}(x, t)$ is the density of the $i$-strategists at location $x$ and time $t$ and $m(x, t)$ is the total density at $x$ and $t\left(m=n_{1}+n_{2}+\cdots+n_{s}\right)$. For simplicity, we drop the $\mathrm{x}$ and $\mathrm{t}$ variables in the equations when there is no ambiguity.

Like the replicator equations, this model assumes that an individual playing strategy $i$ and located at $x$ at time $t$ receives the $a_{i j}$ payoff if it interacts with a neighbor playing strategy $j$, which occurs with a probability approximated by the frequency $n_{i} / m$ of $j$ players at $x$ and $t$. A bookkeeping of the payoff contributions to strategy $i$ from all different $j$ strategies yields the first part of the growth rate of strategy $i$ [the bracketed term in Equations (2)]. In addition there is a regulatory, negative term that accounts for the fact that local densities stay bounded. This regulatory term takes the form of a discount precisely equal to the average payoff earned at location $x$ at time $t$ (which is calculated by summing over $i$ the average payoff to strategy $i$ weighted by its frequency $n_{i} / m$ at location $x$ and time $t$ ). It must be stressed that this discounting term is a very special one, chosen only for mathematical convenience. No particular physiological or behavioral mechanism is known that lets individuals adapt their per capita birth and death rates to local circumstances so as to keep the local population averages of these quantities exactly zero at all times. Cressman and his coworkers (see Cressman and Dash 1987; Cressman 
and Vickers 1997) have elaborated on this issue by assuming that an individual's fitness is composed of its payoff in the contest with other strategies together with a background fitness that is common to all strategies. Their approach has the merit of relating the spatial dynamics of the game to individual life-history traits, but it is hampered by very demanding mathematics (Cressman and Vickers 1997). In Section 3, we take advantage of the great mathematical tractability of replicator-diffusion equations (2) to explore invasion issues in the context of spatial games between cooperative and selfish players. Hutson and Vickers (1995; see also Chapter 22 in Dieckmann et al. 2000) have used the background fitness model of local population regulations to address the same problem. In Section 4 we see that their results are consistent with those obtained through the analysis of the simpler replicator-diffusion equations.

Replicator-diffusion equations form a distinct class of reaction-diffusion models because of their specific reaction term. Equations (2) assume that space is one-dimensional and reduces to an " $x$-axis." The formalism, however, straightforwardly carries over to higher dimensions (see Chapter 22 in Dieckmann et al. 2000 for a detailed account of the rationale of reaction-diffusion models). If the spatial domain is bounded, impermeable boundary conditions are imposed.

\section{Invasibility and evolutionary stability}

A few mathematical results are available to investigate the invasibility or evolutionary stability of a strategy in a spatial game described by replicator-diffusion equations. They all relate the dynamics of the spatial model given by Equations (2) to its nonspatial counterpart, Equations (1). Here, we state the mathematical theorems in a self-contained manner to make them unambiguously applicable to any particular model that falls under their scope. The spatial iterated Prisoner's Dilemma (IPD) offers an opportunity to operate this machinery, as we will see in Section 3.

Vickers (1989) provided the first stability analysis of the replicator-diffusion equations. He found that an interior ESS is so stable that it precludes any spatial dependence:

Proposition 1 If matrix $A$ has an interior ESS, that is, an ESS for the replicator equations [Equations (1)] given by a frequency vector $Q$ with all nonzero coordinates, then this ESS is also stable in the spatial game governed by Equations (2) for all choices of the diffusion coefficients $\mu_{i}$.

The situation becomes much more complicated if there is no interior ESS in the homogeneous game. Hutson and Vickers (1992) addressed the case where there are only two strategies and each pure strategy is an ESS. In the absence of spatial effects each ESS is, by definition, stable. The inclusion of diffusion creates the possibility of a traveling wave that in effect replaces one ESS with the other. To state Hutson and Vickers' main theorem, we first recast the payoff matrix $A$ as

$$
A=\left[\begin{array}{cc}
\alpha & 0 \\
0 & \beta
\end{array}\right]
$$

by setting $\alpha=a_{11}-a_{21}$ and $\beta=a_{22}-a_{12}$, an operation that does not affect the dynamics. To ensure that each pure strategy is an ESS, $\alpha>0$ and $\beta>0$. Hutson and Vickers (1992) investigated the invasion of a region dominated by one strategy - for example, for $x>0$ strategy 1 is played by almost all individuals and for $x<0$ strategy 2 is prevalent.

Proposition 2 Assume that $A$ has the form (3) with $\alpha>0$ and $\beta>0$. There then exists 
a function $F$ such that, if

$$
\frac{\beta}{\alpha}>F\left(\frac{\mu_{1}}{\mu_{2}}\right)
$$

a traveling wave front with positive speed (i.e., moving from $x=-\infty$ to $x=\infty$ along the spatial axis) will connect the two homogeneous pure population equilibria. The function $F$ is well approximated over the range $0.05-20$ by $F(u) \approx u^{-0.61}$.

The following statements explain the importance of Proposition 2 for analyzing applications.

- Proposition 2 states that if inequality (4) is satisfied, then a traveling wave replaces a pure strategy- 1 population with a pure strategy- 2 population. If the inequality is reversed, the sign of the wave speed becomes negative and strategy 2 replaces strategy 1 in a traveling wave. There is virtually no room for coexistence, except perhaps in the atypical boundary case $\beta / \alpha=F\left(\mu_{1} / \mu_{2}\right)$. Thus, in a generic twostrategy game where both strategies are ESSs, a traveling wave necessarily exists and replaces one strategy with the other.

- If the payoffs $\alpha$ and $\beta$ are not influenced by the mobility rates $\mu_{1}$ and $\mu_{2}$, condition (4) asserts that the dominating strategy must have large payoffs and small diffusion rates. (In the IPD, however, the payoffs do depend on the mobility rates.)

- There is strong numerical evidence to support the claim that the propagation of a traveling wave replacing strategy 1 with strategy 2 is strictly equivalent to the growth of a localized clump of individuals playing strategy 2 amid a "sea" of players using strategy 1. Accepting this conjecture, inequality (4) reads as a criterion of invasibility.

While Proposition 1 assumes that the replicator equation admits a stable solution corresponding to an interior (mixed) ESS, Proposition 2 addresses the case where the (ESS) equilibria associated with each pure strategy are the only ones. Vickers et al. (1993) have shed some light on the case where the replicator equation admits an internal stable equilibrium which is not an ESS. This is an interesting case because, in the spatial context, it may lead to the formation of patterns.

Let $\hat{Q}$ be an internal, stable solution to the replicator equation. Assuming that $\hat{Q}$ is not an ESS means that it is invadible, and the simplest situation here arises when $\hat{Q}$ can be invaded by a pure strategy (say, strategy 1). Vickers et al. (1993) proved the following theorem.

Proposition 3 If strategy 1 can invade $\hat{Q}$, that is, $a_{11}>\left(\hat{Q}^{T} A\right)_{1}$, then there exists a combination of mobility rates $\mu_{i}(1 \leq i \leq k)$ such that $\hat{Q}$ is not spatially stable.

This statement is important in light of Turing's (1952) well-known idea that spatial patterns are often associated with equilibria which are stable in the nonspatial system (i.e., without diffusion) and unstable with respect to spatially heterogeneous perturbations. This phenomenon is the so-called Turing instability (see Chapter 22 in Dieckmann et al. 2000). In the framework of replicator-diffusion equations, Vickers et al. (1993) have raised three important points.

- A bifurcation analysis shows that this pattern-formation mechanism is operative in spatial games under the conditions of Proposition 3.

- There must be at least three pure strategies in the game for Proposition 3 to apply. 
- A converse of Proposition 3 holds when there are exactly three strategies. If $\hat{Q}$ resists invasion by any pure strategy, then it is spatially stable and no spatial pattern can be produced.

Patterns arising from the Turing instability vary in space but are constant in time. Yet variations in space and time are essential features of the dynamics of ecological systems. Vickers et al. (1993) have provided a numerical example of a three-strategy game that exhibits another kind of instability (namely, a Hopf bifurcation) that results in spatial patterns which are periodic in time. It should be noted that a general theory of the bifurcations of the three-strategy game is still pending.

\section{Invasion of Tit For Tat in Games with Time-limited Memory}

In Chapter 8 in Dieckmann et al. 2000, Nowak and Sigmund expound on the basics of the IPD game. Here, we refer to concepts and notations introduced by these authors. Investigating the relative invasibility of well-known strategies like the cooperative Tit For Tat (TFT) strategy and the selfish Always Defect $(A D)$ strategy serves to demonstrate some of the mathematical techniques introduced in the previous section.

\section{The spatial struggle of Tit For Tat and Always Defect}

The IPD has proved tremendously fruitful as a paradigm for studying the evolution of cooperation. Game theorists originally identified the TFT strategy as the most robust and stable strategy in the IPD (Axelrod and Hamilton 1981). Subsequent theoretical developments (Nowak and Sigmund 1992, 1993) emphasized that the TFT strategy could be the first step toward cooperation in a world of unconditional defectors playing $A D$. To explain the emergence of cooperation, it is therefore crucial to understand how TFT can gain a foothold in a population dominated by $A D$.

A major problem concerning the nonspatial IPD is that it fails to convincingly settle this issue. Depending on the probability $w$ of continuing the game, either $A D$ is the only ESS, hence TFT has no chance to invade, or both $A D$ and TFT are ESSs (which happens when $w$ is sufficiently large), implying that $T F T$ can invade an established $A D$ population only if the TFT frequency exceeds a certain threshold. Because the nonspatial IPD assumes an infinite population, this result means that an initially finite group of TFT newcomers will never spread. It has long been claimed that small clusters of finite size should still have a chance of spreading, because cooperators within a cluster experience a high probability of interacting with each other. To weigh this claim, one might compare the average payoff earned by a $T F T$ within the cluster with the $A D$ payoff averaged over the whole population. In doing so, however, one would overlook $T F T-A D$ interactions which locally influence the payoff to $A D$ players in the vicinity of the cooperative focus - a local payoff likely to be of critical importance to determining the eventual fate of the TFT population. Numerical examples (Nowak and May 1992, 1993) based on cellular automata demonstrate that local interactions have a significant effect on the outcome of the game between cooperators and defectors. For this reason, there has been much interest in setting up versions of the IPD that specifically account for spatial dynamics and local contests. 


\section{A replicator-diffusion model}

We now assume that TFT and $A D$ players are free to move. We wish to describe the game using a replicator-diffusion equation. This amounts to writing down the payoff matrix $A$ taking into account the organisms' mobility and other individual traits (mortality and interaction time). In the nonspatial game, the parameters are the payoffs $S, P, R, T$ (see Chapter 8 in Dieckmann et al. 2000), and the probability $w$ that two particular interacting individuals continue their interaction in the next round of the game. In the spatial version of the game, that probability $w$ is influenced by the individuals' traits (including the mobility rates), and some work is required to make this relationship explicit.

\section{Microscopic description of interactions.}

We first describe an individual-based model of the population. We assume that each individual in the population occupies a position in space that is a function of time. The population is distributed along a one-dimensional axis: it can be thought of as spread along a coastline or a river bank; or if the environment is really two-dimensional, variations in the strategy mix may occur in one direction only. For the purpose of defining local interactions, we regard space as being divided into discrete contiguous cells of length $\Delta l$ so that each cell contains two individuals at any time. Interactions are initiated between two individuals located in the same cell. Thus $\Delta l$ defines the "interaction length," which we assume to be constant across space. Each interaction lasts $\Delta t$ units of time, which we define as the "interaction time" of the game. Interactions occur consecutively, without any "rest time" in between.

The payoffs $S, P, R$, and $T$ determine the per capita reproductive rate. Thus if the payoff is $S$, for example, to each individual of a group of size $n$, then their numbers increase at a rate $S n$ in the absence of all other effects. We assume that interactions have no direct effect on individual mortality. Let us consider a TFT player within a given cell, and let $p_{T}$ and $p_{D}$ be the probabilities that the partner is a TFT or an $A D$ player, respectively. Then the reproductive success of the nominal TFT player during the small time interval $\Delta t$ is

- $R \Delta t$ if the co-player is a TFT, which occurs with probability $p$;

- $P \Delta t$ if the co-player is a defector already encountered on the previous interaction, which occurs with probability $p_{D} w$;

- $T \Delta t$ if the co-player is a defector not encountered on the previous interaction, which occurs with probability $p_{D}(1-w)$.

Here $w$ denotes the probability that the same two individuals located in a given cell at time $t$ were also sharing a cell at time $t-\Delta t$.

\section{From microscopic interactions to macroscopic dynamics.}

Mobility is modeled by a random walk, and we make the classical diffusion approximation. Thus we define mobility (or diffusion) rates for TFT and $A D$ players, denoted by $\mu_{T}$ and $\mu_{D}$, respectively. Then the derivation of $w$ is straightforward (see Ferrière and Michod 1996, for details) and yields 


$$
\begin{aligned}
w= & {\left[4 \sqrt{\pi} \sqrt{\left(\mu_{T}+\mu_{D}\right) \Delta t}\right]^{-1} } \\
& \times \int_{u, v \in[-\Delta l / 2, \Delta l / 2]} \exp \left[-\frac{(u-v)^{2}}{4\left(\mu_{T}+\mu_{D}\right) \Delta t}\right] d u d v .
\end{aligned}
$$

For small cell length $\Delta l$, the following approximation holds [Equation (7) in Ferrière and Michod 1996]:

$$
w \approx \frac{2}{\sqrt{\pi}} \frac{\Delta l}{\sqrt{\Delta t}} \frac{\mu_{T} \mu_{D}}{\left(\mu_{T}+\mu_{D}\right)^{5 / 2}} .
$$

We derive a replicator-diffusion model of the population dynamics by letting $\Delta l$ go to zero and rescaling time appropriately, such that $\Delta l / \sqrt{\Delta t}$ approaches a positive constant $v$ :

$$
\frac{\Delta l}{\sqrt{\Delta t}} \rightarrow v \neq 0
$$

Now we can define the densities of $T F T$ and $A D$ as continuous functions of space and time, denoted by $n_{T}(x, t)$ and $n_{D}(x, t)$. Let $m$ be the total density $n_{T}+n_{D}$. We have $p_{T}=n_{T} / m, p_{D}=n_{D} / m$; thus, the TFT reproductive rate is

$$
\frac{n_{T}}{m} R+\frac{n_{D}}{m}[w P+(1-w) S] .
$$

Likewise, the $A D$ reproductive rate is

$$
\frac{n_{D}}{m} P+\frac{n_{T}}{m}[w P+(1-w) T] .
$$

The payoff matrix of the replicator-diffusion game equations (2) follows readily:

$$
A=\left[\begin{array}{cc}
R & w P+(1-w) S \\
w P+(1-w) T & P
\end{array}\right],
$$

with $w$ given by

$$
w=\frac{2 v}{\sqrt{\pi}} \frac{\mu_{T} \mu_{D}}{\left(\mu_{T}+\mu_{D}\right)^{5 / 2}} .
$$

Notice that for consistency with the assumption made above - that the interaction length is constant across space - the total density should vary very slowly in time and smoothly across space. Using numerical integration of Equation (2) with $A$ given by Equations (10) and (11), we have found this requirement to be fulfilled when $\mu_{T}$ and $\mu_{D}$ were not vastly different.

\section{A spatial version of Hamilton's rule}

To analyze the replicator-diffusion model of the IPD by means of the theory developed in Section 2, we must first consider the nonspatial version of the system and investigate its equilibria. With this aim in view, it is convenient to introduce a cost-benefit parameterization of the IPD payoffs (Brown et al. 1982). Assume that a cooperator exhibits some behavior that benefits the fitness of its partner, the recipient, by an amount $b$ which is larger than 0. The benefit is independent of the recipient's behavior. By providing 


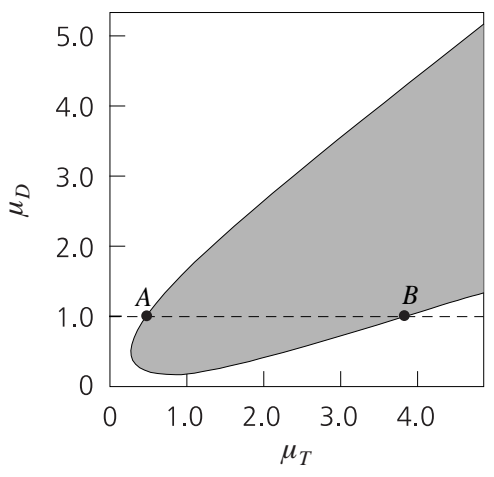

Figure 1 Mobility rates leading to an invasion of an established $A D$ population by a wave of $T F T$ players. The model assumes time-limited memory and is given by the system of replicator-diffusion equations (2). The payoff matrix $A$ is specified by Equations (10) and (11) with $v=1$. The shaded area contains all pairs of mobility rates $\mu_{T}, \mu_{D}$ such that the coefficient of reciprocation $H\left(\mu_{T}, \mu_{D}\right)$ is larger than the costbenefit ratio $c / b$ (fixed at 0.22 ). For given defectors' mobility $\mu_{D}$ larger than a minimum value $(\approx 0.11)-$ for example, $\mu_{D}=1.0$ (dashed line) - there is an interval $(A, B)$ of $\mu_{T}$ mobility rates over which a TFT invading wave displaces a resident $A D$ population.

its partner with the benefit $b$, the cooperator incurs a cost $-c, c>0$. Again, this cost is independent of the recipient's behavior. If the effects on fitnesses are additive, with a baseline value taken to be 1 , one obtains the following parameterization: $T=1+b$, $R=1+b-c, P=1, S=1-c$.

Using this parameterization, we see that the replicator equation of the game admits two stable equilibria (corresponding to each pure strategy) whenever

$$
w \geq \frac{c}{b}
$$

which is the condition found by Brown et al. (1982) for TFT and AD to simultaneously be ESSs in the standard, nonspatial game. Then Proposition 2 asserts that there exists a traveling wave replacing $A D$ with $T F T$ if

$$
\frac{w}{1+(1-w) F\left(\mu_{T} / \mu_{D}\right)}>\frac{c}{b} \text {. }
$$

This inequality provides a Hamilton rule (Hamilton 1964) for the increase of cooperation in a nonsocial, spatial environment. The left-hand side (hereafter denoted by $H$ ) generalizes the coefficient of reciprocation defined for the nonspatial IPD (Brown et al. 1982), which gives the probability that an individual's cooperative act is returned via reciprocation from other TFT. The right-hand side of the inequality is the cost-benefit ratio of cooperation. This spatial Hamilton rule can be further extended to include a cost to mobility (Ferrière and Michod 1996).

Inequality (13) defines a set of mobility rates $\mu_{T}$ and $\mu_{D}$ that cause an invasion of defectors $A D$ by $T F T$ : a traveling wave replaces $A D$ with $T F T$. All other parameters being fixed, this set is delineated by the $c / b$ isoclines drawn on the surface $H\left(\mu_{T}, \mu_{D}\right)$ (see Figure 1). [Notice that if a pair $\mu_{T}, \mu_{D}$ satisfies inequality (13), then it automatically meets inequality (12).] We find that a range of mobility rates exists for which TFT can invade provided that $A D$ mobility exceeds a minimum threshold. In general, this range includes the mobility rate of resident defectors, $\mu_{D}$, but it is skewed around $\mu_{D}$ so that TFT players may be much more mobile than defectors and yet successfully displace them.

As a consequence of the particular form taken by the function $F$ in Equation (13) $\left[F(u) \approx u^{-0.61}\right.$, see Proposition 2], a condition for the invasion by rare $A D$ s of a TFT population is obtained by reversing inequality (13). (Note that the particular form of $F$ is 
only an approximation. Dealing with the exact function would call for further investigation.) This condition determines the stability of TFT once established. It turns out that TFT is jeopardized by $A D$ endowed with either high or very low mobility (see Figure 1). Also, TFT is immune to invasion for a much wider range of $A D$ mobility rates when its own rate of mobility increases. Thus, by moving at higher rates, cooperators find more efficient protection against reinvasion by $A D$.

\section{Why mobility can favor Tit For Tat}

To answer this question and to give some intuitive understanding of the above results, Ferrière and Michod (1995) have developed an auxiliary model focusing on the stochastic motion of the players. Heuristically, the growth of an initially small cluster relies on two conditions: first, that the cluster can spread outward from the edge, and, second, that its core is not destroyed by $A D$ intruders (Axelrod 1981; Eshel and Cavalli-Sforza 1982; Wilson et al. 1992). The first condition is ensured whenever TFTs can make safe moves toward the front of the invasion, that is, whenever TFT pioneers can avoid being suckered as they move outward. A TFT pioneering to the front of an invasion will not be suckered there if it can get assorted with another TFT also on a pioneering move or if it moves together with a known $A D$ (in which case it will retaliate). The auxiliary model set up by Ferrière and Michod (1995) shows that both conditions are more likely to be met for high (but not too high) mobility in TFT and $A D$. Likewise, the second condition is met if a defector entering the core of a TFT cluster gets assorted there with another $A D$ or if it undergoes retaliation by a TFT also moving back to the core. Again, the likelihood that either case will be realized is maximized at high TFT and $A D$ mobility rates. To summarize, the following events are crucial for the emergence of $T F T$ and are enhanced by significant mobility of the players: assortative meetings of TFTs at the front of an invasion or of $A D \mathrm{~s}$ in the core of the cluster, and tracking of $A D \mathrm{~s}$ by TFTs toward the front or toward the core.

\section{Invasion of Tit For Tat in Games with Space-limited Memory}

There are two important assumptions underlying the IPD replicator-diffusion equations investigated in the previous section. First, the memory is "space-extended" but "timelimited." That is, a player can recognize its opponent wherever they meet, but the player's memory is limited to the last round of the game. Second, the local density of each strategy is assumed to vary very slowly, in agreement with the assumption made in the microscopic description of the population that the spatial axis can be divided into contiguous cells of constant length, each cell containing two individuals. Hutson and Vickers (1995) have developed a different reaction-diffusion model of the spatial IPD where these assumptions have been modified or relaxed. In the Hutson-Vickers model, memory is not restricted to the last interaction but instead is space-limited: a player can remember any of its previous opponents provided that neither has moved out of the cell where they first met. Furthermore, a cell may now contain a variable number of players. The goal of this section is to present some important results drawn from their approach after examining structural differences between this model and the previous game-diffusion equations. 


\section{Model description}

The Hutson-Vickers model is fully expounded in Chapter 22 in Dieckmann et al. 2000 (Section 22.2, A model for invasion of Tit For Tat). Here, we content ourselves with highlighting the specificities of this model.

- Local interactions. The spatial axis is still divided into contiguous cells of constant length $l$, but now each cell may contain many individuals. Opponents of any player in a given cell are drawn randomly within that cell.

- Repeated interactions. The Hutson-Vickers model relaxes the assumption that local population size varies on a slow time scale. Consequently, the probability $w$ of players meeting is no longer a constant. In their model, Hutson and Vickers (1995) recast $w$ into a dynamic "getting-to-know" function [denoted by $g(x, t)$ ] that gives the proportion of $A D$ (or $T F T$ ) players within a cell that a typical $T F T$ (or $A D$ ) player has already met. They further define $G=g n_{T} n_{D}$ as the number density of $T F T-A D$ pairs within a cell that have already met.

- Memory. Memory is not limited to the last round. A TFT player recognizes an opponent on a second or subsequent occasion provided that neither has left the cell where the encounter occurred. This is in contrast with the game-diffusion model, where recognition may occur wherever the encounter takes place, but only on the next interaction.

- Population regulation. The per capita death rate is made density dependent. Therefore, it varies in space and time (but, as before, it is not influenced by the outcome of the game).

\section{Main properties of the model}

The analysis of the Hutson-Vickers model stems from ideas similar to those underlying Proposition 2. First, one has to determine the possible population equilibria assuming that player densities are spatially homogeneous. One may then turn to the effect of locally perturbing the stable equilibria, thereby mimicking the effect of an invasion attempt. When players have spatially homogeneous distributions, their densities and the gettingto-know function depend only on $t$. Then the model reduces to a system of ordinary differential equations [set $\partial^{2} u / \partial x^{2}=0$ and $\partial^{2} v / \partial x^{2}=0$ in Equations (22.17) and replace all partial derivatives with respect to $t$ with ordinary derivatives in Equations (22.17) and (22.19) of Chapter 22 in Dieckmann et al. 2000]. Standard techniques of local stability analysis can now be used. The system turns out to have one of three simple structures:

1. There are stable equilibria with only TFT-players and only $A D$-players, and an unstable coexistence equilibrium.

2. There is a stable pure $A D$ state and the only other equilibrium, that with just $T F T$ players, is unstable.

3. In addition to the equilibria of structure (2), there are two coexistence states (one stable and one unstable) of TFT and $A D$.

The next step aims at determining whether a pure TFT state may evolve starting from initial conditions where TFT players are localized within an established $A D$ population. 


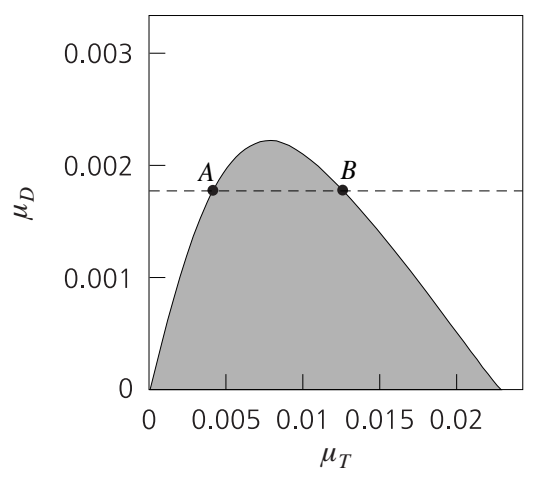

Figure 2 Mobility rates leading to an invasion of an established $A D$ population by a wave of $T F T$ players. The model assumes space-limited memory and can be written as a system of reaction-diffusion equations [see Chapter 22 in Dieckmann et al. 2000, Equations (22.17) and (22.19)]. The shaded area contains all pairs of mobility rates $\mu_{T}, \mu_{D}$ for which there exists a traveling wave replacing $A D$ with TFT. For given defectors' mobility $\mu_{D}$ smaller than a maximum value $(\approx 0.0021)$ - for example, $\mu_{D}=0.0018$ (dashed line) - there is an interval $(A, B)$ of $\mu_{T}$ mobility rates over which invasion by TFT occurs. Model parameters: $\alpha=1.3725, \beta=0.9, \gamma=1.45, \delta=1, k=4, b=0=d, \sigma=1, \theta=100\left(\mu_{T}+\mu_{D}\right)$.

This requires that the homogeneous TFT state must be stable, which actually happens with structure (1). Thus, we must deal with a situation similar to that handled by Proposition 2: two stable states and traveling waves that may "connect" them. However, the Hutson-Vickers model is not written as a system of replicator-diffusion equations (hence Proposition 2 does not apply), and a theoretical treatment presents rather formidable difficulties. A computational study suffices, however, to demonstrate the remarkable richness of the model's behavior. The most noteworthy point, as illustrated by the numerical example presented hereafter, is that large or small players' mobilities cannot be claimed to be unambiguously good or bad for the evolution of cooperation.

\section{Existence of invading waves of Tit For Tat}

In contrast with the case of replicator-diffusion models, no general theorem is available to guarantee the existence of traveling wave solutions to Hutson-Vickers equations. Yet numerical procedures do provide evidence that invasion dynamics develop wave patterns. As in Proposition 2, the sign of the wave speed determines whether the wave replaces $A D$ with $T F T$, or $T F T$ with $A D$. Figure 2 shows how for a specific set of parameter values the mobility rates influence the outcome of the game. To a certain extent, the results confirm those of the previous section. For small or large $\mu_{T}$ the cooperators are defeated, whereas for medium $\mu_{T}$ TFT successfully invades.

Hutson and Vickers (1995) gave the following interpretation of this result. Consider what happens as $\mu_{T}$ is reduced at points $A$ and $B$ in Figure 2. These points indicate stalemates, that is, traveling waves with zero speed. When $\mu_{T}$ is reduced, the wave front of the TFT players steepens so that density is reduced in the leading edge of the wave. The key factor is that at $A$ the number of encounters between any given two players is determined by the death rate, whereas at $B$ it is determined by mobility. At $B$, the getting-to-know function $g$ and the number of $A D-T F T$ pairs that have already met $G$ increase significantly, causing the per capita birth rate in TFT to increase. Thus the TFT players have the advantage and a wave develops in which they advance. By contrast, at $A$ there is little change in functions $g$ and $G$ so that the per capita birth rate of TFT is mainly influenced by the decrease in $T F T$ density, which gives $A D$ the advantage. The same type of argument suggests that reducing $\mu_{D}$ is always bad for $A D$, which is consistent 
with Figure 2 but contrasts with conclusions drawn from the replicator-diffusion model.

\section{Concluding Comments}

The importance of spatial structure for the IPD has long been realized (see, e.g., Axelrod 1984). Intuitively, individual mobility in the IPD is expected to raise an insurmountable obstacle to the spread of cooperation by allowing egoists to exploit cooperativeness and escape retaliation (Houston 1993). Dugatkin and Wilson (1991) and Enquist and Leimar (1993) addressed the issue, but their models had several limitations: only $A D$ players were mobile; mobility was represented implicitly through some traveling cost; and only the question of the stability of TFT against $A D$ was considered.

Reaction-diffusion models offer a natural framework to incorporate temporal and spatial effects in games. These models represent players that move in space in a random manner at a rate controlled by specific parameters. Their development is rooted in the Taylor--Jonker replicator equations (1). Players' mobility is included through the standard diffusion approximation of spatial motion, which yields second-order derivatives with respect to the spatial variable in Equations (2). We call the resulting system a "replicatordiffusion model." The reaction term can be modified further to allow for population limitation through density-dependent payoffs (Cressman and Dash 1987).

Once the reaction-diffusion model has been set up, one can address the central question in game theory: can an established population of one or several strategies be invaded by an initial spatially limited distribution of individuals playing an alternative strategy? Propositions 1 to 3 provide some insights into this problem in the context of replicator-diffusion models. The spatial dimension does not affect the stability of an internal strategy mix (i.e., all strategies are represented), which is an ESS in the standard game (Proposition 1). When there are only two strategies and both are ESSs in the nonspatial game, space dramatically alters the picture by allowing one strategy to displace the other (Proposition 2). Finally, in games with three (or more) strategies, spatial patterns (that is, spatially heterogeneous but temporally "frozen" distributions of coexisting strategies) develop when the replicator equation possesses a stable internal equilibrium that is not an ESS (Proposition 3). These results have been extended to spatial games including logistic population regulation (Cressman and Vickers 1997).

From the point of view of finding explicit, tractable invasibility criteria, two-strategy replicator-diffusion models are quite remarkable. If there is only one pure ESS in the standard game or if there is a mixed ESS, the stability property carries over nicely to the spatial game. A difficulty arises when both pure strategies are ESSs in the nonspatial game. In the spatial setting, the mathematical theory (Hutson and Vickers 1992) offers three statements that constitute the core of Proposition 2: one strategy invades and replaces the other (no coexistence); the invasion dynamics develop as a traveling front; there is a clear-cut invasibility criterion based on the sign of the speed of the traveling wave. On the basis of numerical simulations, the same invasibility rule proves to also apply to the more involved Hutson-Vickers model. Therefore, in these models it is the emergence of traveling waves that determines the evolutionary fate of individuals. The wave acts as a "vehicle" for population conflict (which mainly occurs around the fringe of the wave). In a sense, selection operates "at the level of the wave," although the wave itself is not a self-reproducing unit, just an expanding one. Obviously, the properties of waves are not in the definition of the system, instead they are derived from the individuals' behavioral and demographic traits. A similar phenomenon has been observed in individual-based models of host-parasitoid interactions where the formation of spiral waves determine the invasion success of mutant parasitoids (Boerlijst et al. 1993). 
Other versions of the spatial IPD, designed as cellular automata, have recently been issued (Lindgren and Nordahl 1994; Nakamaru et al. 1997; see Chapter 13 in Dieckmann et al. 2000). Differences between these models and the reaction-diffusion approach lie in various (biological) assumptions about individual mobility and the effect of the game on individual life histories. The game pay-offs translate into a transmission rate (i.e., the probability of invading a neighboring site) in the model designed by Lindgren and Nordahl (1994), whereas they determine mortality rates in the framework by Nakamaru et al. (1997). The former model was analyzed though computer simulations; the latter received an analytical treatment by means of pair-approximation techniques (see Chapters 13 and 18 in Dieckmann et al. 2000). In both models, mobility is restricted to the dispersal of one offspring into a vacant neighboring site. Consequently, neither model allows connections to be drawn between the outcome of the game and different levels of individual mobility. Van Baalen and Rand (1998) have also developed a pair-approximation model of competition between altruists and non-altruists in a viscous population, in which they incorporated a rate of mobility (the same for both types of individuals). Although their system is not an iterated game, there is an interesting parallel between its behavior and that of the replicator-diffusion model. Again, invasion appears to be governed by a "spatially extended" Hamilton rule, where the coefficient of relatedness is recast into a coefficient of reciprocation depending on the birth, death, and mobility rates - much like the left-hand side of Equation (13). Also, the unit of selection becomes a "characteristic cluster" whose structure is described by a stable distribution of pairs of neighboring site occupancies, altruist-altruist, altruist-selfish, altruist-empty (a distribution that can be calculated from the model parameters). Van Baalen and Rand's model predicts that altruism can invade a selfish population background provided that the individual mobility rate is close to some optimum, intermediate value. As in the reaction-diffusion models, this ensures that the "scale of dispersal" is larger than the "scale of interaction." In other words, dispersal should be limited to guarantee a sufficient proportion of altruist-altruist pairings, but strong enough to ensure that altruists can "export" themselves and propagate through the environment.

The issue of invasion in spatial games arises from the study of a fascinating biological enigma - the origin and maintenance of cooperation - and yields profound mathematical challenges. The key relation between the existence of a traveling wave and invasion from a localized cluster is widely accepted on the basis of overwhelming numerical simulations; however, it has yet to be proved mathematically (see Chapter 22 in Dieckmann et al. 2000). The most urgent issue might be to further probe how the local mean-field description of spatial games based on reaction-diffusion models departs from the dynamics of the underlying discrete system of interacting individuals. Individual models cannot reach a sufficient level of generality, nor do they succeed at pointing out details at the individual level that are critical for understanding the macroscopic dynamics. Intermediate descriptions - for example, through moment or correlation equations (see Chapters 18 to 21 in Dieckmann et al. 2000) - have yet to be improved with respect to dealing with the initial stages of invasion processes, when the invading population is limited to a small area in space. In the meantime, we believe that the models of spatial games described in this chapter represent a significant improvement over previous mathematical attempts to describe the IPD and explain the evolution of cooperation. 


\section{References}

Axelrod R (1981). The emergence of cooperation among egoists. American Political Science Review 75:306-318

Axelrod R (1984). The Evolution of Cooperation. New York, NY, USA: Basic Books

Axelrod R, Hamilton WD (1981). The evolution of cooperation. Science 211:1390-1396

Boerlijst M, Lamers ME, Hogeweg P (1993). Evolutionary consequences of spiral waves in a hostparasitoid system. Proceedings of the Royal Society of London B 253:15-18

Brown J, Sanderson M, Michod R (1982). Evolution of social behavior by reciprocation. Journal of Theoretical Biology 99:319-339

Cressman R, Dash A (1987). Density dependence and evolutionarily stable strategies. Journal of Theoretical Biology 126:393-406

Cressman R, Vickers G (1997). Spatial and density effects in evolutionary game theory. Journal of Theoretical Biology 184:359-369

Dieckmann U (1997). Can adaptive dynamics invade? Trends in Ecology and Evolution 12:128-131

Dieckmann U, Law R, Metz JAJ, eds. (2000). The Geometry of Ecological Interactions: Simplifying Spatial Complexity. Cambridge, UK: Cambridge University Press

Diekmann O, Christiansen F, Law R (1996). Evolutionary dynamics, Editorial. Journal of Mathematical Biology 34:483

Dugatkin L, Wilson D (1991). Rover: A strategy for exploiting cooperators in a patchy environment. The American Naturalist 138:687-701

Enquist M, Leimar O (1993). The evolution of cooperation in mobile organisms. Animal Behaviour 45:747-757

Eshel I, Cavalli-Sforza L (1982). Assortment of encounters and the evolution of cooperativeness. Proceedings of the National Academy of Sciences of the USA 79:1331-1335

Ferrière R, Michod R (1995). Invading wave of cooperation in a spatial iterated Prisoner's Dilemma. Proceedings of the Royal Society of London B 259:77-83

Ferrière R, Michod R (1996). The evolution of cooperation in spatially heterogeneous populations. The American Naturalist 147:692-717

Geritz S, Metz J, Kisdi E, Mesézna G (1997). The dynamics of adaptation and evolutionary branching. Physical Review Letters 78:2024-2027

Hadeler K (1981). Diffusion in Fisher's population model. Rocky Mountain Journal of Mathematics 11:39-45

Hamilton WD (1964). The genetic evolution of social behavior. Journal of Theoretical Biology $7: 1-52$

Hofbauer J, Sigmund K (1998). Evolutionary Games and Population Dynamics. Cambridge, UK: Cambridge University Press

Houston A (1993). Mobility limits cooperation. Trends in Ecology and Evolution 8:194-196

Hutson V, Vickers GT (1992). Travelling waves and dominance of ESSs. Journal of Mathematical Biology 30:457-471

Hutson V, Vickers, GT (1995). The spatial struggle of Tit-For-Tat and defect. Philosophical Transactions of the Royal Society of London B 348:393-404

Lindgren K, Nordahl MG (1994). Evolutionary dynamics of spatial games. Physica D 75:292-309

Maynard Smith J (1982). Evolution and the Theory of Games. Cambridge, UK: Cambridge University Press

Metz JAJ, Nisbet R, Geritz S (1992). How should we define "fitness" for general ecological scenarios? Trends in Ecology and Evolution 7:198-202

Nakamaru M, Matsuda H, Iwasa Y (1997). The evolution of cooperation in a lattice-structured population. Journal of Theoretical Biology 184:65-81

Nowak MA, May RM (1992). Evolutionary games and spatial chaos. Nature 359:826-829

Nowak MA, May RM (1993). The spatial dilemmas of evolution. International Journal of Bifurcation and Chaos 3:35-78

Nowak MA, Sigmund K (1992). Tit For Tat in heterogeneous populations. Nature 355:250-253

Nowak MA, Sigmund K (1993). A strategy of win-stay, lose-shift that outperforms Tit-for-Tat in the Prisoner's Dilemma game. Nature 364:56-58

Taylor P, Jonker L (1978). Evolutionarily stable strategies and game dynamics. Mathematical 


\section{Biosciences 40:145-156}

Trivers R (1971). The evolution of reciprocal altruism. Quarterly Review of Biology 46:35-57

Turing A (1952). The chemical basis of morphogenesis. Philosophical Transactions of the Royal Society of London B 237:37-72

van Baalen M, Rand DA (1998). The unit of selection in viscous populations and the evolution of altruism. Journal of Theoretical Biology 193:631-648

Vickers GT (1989). Spatial patterns and ESSs. Journal of Theoretical Biology 140:129-135

Vickers GT, Hutson V, Budd C (1993). Spatial patterns in population conflicts. Journal of Mathematical Biology 31:411-430

Wilson DS, Pollock GB, Dugatkin LA (1992). Can altruism evolve in purely viscous populations? Evolutionary Ecology 6:331-341

Zeeman E (1980). Population dynamics from game theory. In Global Theory of Dynamical Systems. Lecture Notes in Mathematics 819, ed. Nitecki A, Robinson C, pp. 471-497. Berlin, Germany: Springer 